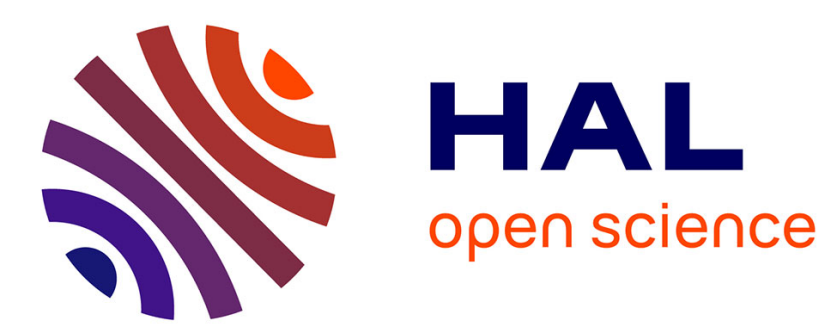

\title{
A new subgrid eddy-viscosity model for large-eddy simulation of anisotropic turbulence
}

\author{
Guixiang X. Cui, Chun-Xiao Xu, Le Fang, L. Shao, Z.S. Zhang
}

\section{To cite this version:}

Guixiang X. Cui, Chun-Xiao Xu, Le Fang, L. Shao, Z.S. Zhang. A new subgrid eddy-viscosity model for large-eddy simulation of anisotropic turbulence. Journal of Fluid Mechanics, 2007, 582, pp.377-397. 10.1017/S002211200700599X . hal-00272158

\section{HAL Id: hal-00272158 \\ https://hal.science/hal-00272158}

Submitted on 14 Jan 2010

HAL is a multi-disciplinary open access archive for the deposit and dissemination of scientific research documents, whether they are published or not. The documents may come from teaching and research institutions in France or abroad, or from public or private research centers.
L'archive ouverte pluridisciplinaire HAL, est destinée au dépôt et à la diffusion de documents scientifiques de niveau recherche, publiés ou non, émanant des établissements d'enseignement et de recherche français ou étrangers, des laboratoires publics ou privés. 


\title{
A new subgrid eddy-viscosity model for large-eddy simulation of anisotropic turbulence
}

\author{
G. X. CUI $I^{1, \dagger}$, C. X.XU ${ }^{1}$, L. FANG ${ }^{1,2}$, L. SHAO ${ }^{2}$ AND Z. S. ZHANG ${ }^{1}$ \\ ${ }^{1}$ Department of Engineering Mechanics, Tsinghua University, Beijing, China \\ ${ }^{2}$ Laboratory of Fluid Mechanics and Acoustics, Ecole Centrale de Lyon, France
}

(Received 8 November 2005 and in revised form 25 January 2007)

A new subgrid eddy-viscosity model is proposed in this paper. Full details of the derivation of the model are given with the assumption of homogeneous turbulence. The formulation of the model is based on the dynamic equation of the structure function of resolved scale turbulence. By means of the local volume average, the effect of the anisotropy is taken into account in the generalized Kolmogorov equation, which represents the equilibrium energy transfer in the inertial subrange. Since the proposed model is formulated directly from the filtered Navier-Stokes equation, the resulting subgrid eddy viscosity has the feature that it can be adopted in various turbulent flows without any adjustments of model coefficient. The proposed model predicts the major statistical properties of rotating turbulence perfectly at fairly low-turbulence Rossby numbers whereas subgrid models, which do not consider anisotropic effects in turbulence energy transfer, cannot predict this typical anisotropic turbulence correctly. The model is also tested in plane wall turbulence, i.e. plane Couette flow and channel flow, and the major statistical properties are in better agreement with those predicted by DNS results than the predictions by the Smagorinsky, the dynamic Smagorinsky and the recent Cui-Zhou-Zhang-Shao models.

\section{Introduction}

Large-eddy simulation is believed to be a potential method for numerical prediction of complex turbulent flows (Jimenez \& Moser 2000; Pope 2004). However, a number of numerical and physical issues must be resolved before it can be properly used in practice when the computer resources are available. This paper focuses on the physics side, i.e. the subgrid stress model of large-eddy simulation. A number of subgrid stress models are already available, but none of them is fully satisfactory. For instance, the Smagorinsky model (Smagorinsky 1963) is over-dissipative and the scale similarity model is under-dissipative (Bardina, Ferziger \& Reynolds 1987). The spectral eddy-viscosity model based on EDQNM theory (Chollet and Lesieur 1981) is good for homogeneous turbulence, but it cannot be used in complex turbulent flows. The so-called dynamic subgrid model (Gemano et al. 1991) is now popularly used in numerical prediction of complex turbulent flows in combination with a reference model. The dynamic model is essentially a dynamic procedure for determining the model coefficient and it cannot remove artefacts in the reference model. Nicoud \& Ducros (1999) and Vreman (2004) reformulated subgrid eddy viscosity with

$\dagger$ Author to whom correspondence should be addressed: demzzs@mail.tsinghua.edu.cn; cgx@ mail.tsinghua.edu.cn. 
consideration of the asymptotic behaviour of velocity fluctuations in the near-wall region and used the velocity gradient tensor in the reconstruction of subgrid eddy viscosity. These models remove the over-dissipation of the Smagorinsky model in the near-wall regions. Detailed discussions of the subgrid stresses (SGS) model can be found in Sagaut (2002) and Guerts (2004).

In this paper, we consider the eddy-viscosity-type model of SGS since it is easily accessible to Navier-Stokes solver. The essence of eddy viscosity is the energy transfer between resolved and unresolved scale turbulence. Some authors (e.g. Jimenez 2000; Pope 2004) emphasize the importance of the energy dissipation property of the subgrid model rather than the momentum transfer property, and they explain why the Smagorinsky model is better than the scale similarity model in prediction of turbulent shear flow, although the former is phenomenological and must tune the model coefficient, e.g. dynamic Smagorinsky model. Most of the available subgrid eddyviscosity models are of the phenomenological type and do not consider the kinetic energy transfer of anisotropic turbulence explicitly in the formulation of SGS models. In this paper, we focus on the effect of anisotropy on the subgrid eddy viscosity with consideration of energy transfer between revolved and unresolved scale turbulence.

It is possible to construct a subgrid eddy-viscosity model with consideration of turbulent kinetic energy transfer between resolved and unresolved scale turbulence from the Navier-Stokes equation. For instance, Meneveau (1994) proposed an eddyviscosity model by means of the Kolmogorov equation for filtered velocity in isotropic turbulence. Cui et al. (2004, hereinafter referred to as CZZS) formulated a detailed eddy-viscosity model with a similar idea to that of Meneveau and used the model satisfactorily to predict isotropic turbulence and turbulent channel flows. However, the CZZS model failed to predict strongly anisotropic turbulence, e.g. rotating turbulence at low turbulent Rossby number, $u_{r m s} / \Omega \lambda$, in which $u_{r m s}$ is the root mean square of velocity fluctuations, $\Omega$ is the rotating rate and $\lambda$ is the Taylor micro scale. In this paper, a local volume-averaged Kolmogorov equation is derived for resolved scale turbulence and used for formulating the subgrid eddy-viscosity model. The eddy viscosity is assumed to be a scalar; hence, it is an isotropic model. However, the new model takes into account the anisotropic effects in the energy transfer between resolved and unresolved scale turbulence by means of the local volume average of the structure function equation in displacement space. The new model is tested in homogeneous rotating turbulence, plane turbulent Couette flow and plane turbulent channel flow. The results show that the new model is able to predict major characteristics of rotating turbulence at fairly low Rossby number, i.e. high rotation rate. For plane turbulent Couette flow and channel flow, the new model produces a better prediction than the Smagorinsky model, dynamic Smagorinsky model and CZZS model.

In this paper, full details of the formulation of the new model are presented in $\S 2$. In $\S 3$, the predicted results for rotating turbulence will be addressed with analyses and $\S 4$ shows the computational results of plane turbulent Couette flow and channel flow. Discussions and concluding remarks are given in $\S 5$.

\section{Formulation of the subgrid eddy-viscosity model for homogeneous anisotropic turbulence}

As mentioned in $\S 1$, the new eddy-viscosity model is based on the local volumeaveraged generalized Kolmogorov equation. The previous version of this type of model is under the assumption of homogeneity and isotropy of turbulence (Meneveau 1994; Cui et al. 2004). In this paper, homogeneity of turbulence is still assumed, but 
turbulence is anisotropic. To take into account anisotropy of homogeneous turbulence in turbulent kinetic energy transfer, we derived the Kolmogorov equation directly from the Navier-Stokes equation. Two typical anisotropic turbulent flows are considered, namely the homogeneous shear turbulence and homogeneous rotating turbulence. The governing equations for homogeneous shear turbulence are written in the inertial frame as follows

$$
\begin{gathered}
\frac{\partial V_{i}}{\partial t}+V_{k} \frac{\partial V_{i}}{\partial x_{k}}=-\frac{1}{\rho} \frac{\partial p}{\partial x_{i}}+v \frac{\partial^{2} V_{i}}{\partial x_{k} \partial x_{k}}, \\
\frac{\partial V_{i}}{\partial x_{i}}=0 .
\end{gathered}
$$

In (2.1), (2.2) and hereinafter, the Einstein convention is used for repeated indexes. To derive the subgrid model in homogeneous turbulence, we decompose the velocity into a mean and fluctuations

$$
V_{i}=U \delta_{i 1}+u_{i}
$$

in which $u_{i}$ is the fluctuations and $U$ is the ensemble-averaged velocity in the $x_{1}$ direction. For homogeneous shear flow, $U=\gamma x_{2}$ where $\gamma$ is the constant mean shear rate. The equation for the fluctuations can be derived readily from (2.1) and (2.2) sach that

$$
\begin{aligned}
\frac{\partial u_{i}}{\partial t}+U \frac{\partial u_{i}}{\partial x_{1}}+u_{k} \frac{\partial u_{i}}{\partial x_{k}} & =-\frac{1}{\rho} \frac{\partial p}{\partial x_{i}}+v \frac{\partial^{2} u_{i}}{\partial x_{k} \partial x_{k}}+s_{i}, \\
\frac{\partial u_{i}}{\partial x_{i}} & =0 .
\end{aligned}
$$

In (2.4), a source term, i.e. $s_{i}=-\gamma u_{2} \delta_{i 1}$, on the right-hand side is added as an external force term for the fluctuating motion.

For homogeneous rotating turbulence, it is necessary to write the Navier-Stokes equation in the rotating frame which is naturally used in computing rotating flows, e.g. in turbo machinery or atmosphere. In homogeneous rotating turbulence, the mean velocity can be set to zero and the governing equations of velocity fluctuations can be written as follows

$$
\begin{gathered}
\frac{\partial u_{i}}{\partial t}+u_{k} \frac{\partial u_{i}}{\partial x_{k}}=-\frac{1}{\rho} \frac{\partial p}{\partial x_{i}}+v \frac{\partial^{2} u_{i}}{\partial x_{k} \partial x_{k}}+2 \varepsilon_{i k 3} \Omega u_{k}, \\
\frac{\partial u_{i}}{\partial x_{i}}=0 .
\end{gathered}
$$

In (2.6), rotation is in the $x_{3}$-direction and $\varepsilon_{i k 3}$ is a component of the permutation tensor $\varepsilon_{i k j}$ with $j=3$. In (2.6), the source term $\varepsilon_{i k 3} \Omega u_{i}$ is the Coriolis force and the pressure term is equal to hydrodynamic pressure plus the potential of the centrifugal force.

The governing equations for both homogeneous rotating turbulence and shear turbulence can be written in a unified form for convenient derivation of subgrid eddy viscosity as follows

$$
\begin{aligned}
\frac{\partial u_{i}}{\partial t}+U \frac{\partial u_{i}}{\partial x_{1}}+u_{k} \frac{\partial u_{i}}{\partial x_{k}} & =-\frac{1}{\rho} \frac{\partial p}{\partial x_{i}}+v \frac{\partial^{2} u_{i}}{\partial x_{k} \partial x_{k}}+s_{i}, \\
\frac{\partial u_{i}}{\partial x_{i}} & =0
\end{aligned}
$$


in which $U=\gamma x_{2}, s_{i}=-\gamma u_{2} \delta_{i 1}$ for homogeneous shear turbulence, and $U=0, s_{i}=$ $2 \varepsilon_{i k 3} \Omega u_{k}$ in homogeneous rotating turbulence. It should be emphasized that the reference frames for rotating turbulence and shear turbulence are different, although their governing equations are written in same formulae. This indicates that the subgrid eddy viscosity for rotating turbulence is reconstructed in a rotating frame whereas it is in an inertial frame for homogeneous shear turbulence.

Filtering (2.8) and (2.9), we can obtain the governing equation for large-eddy simulation (LES). Note that LES of rotating turbulence should consider the transformation property (Horiuti 2006) between rotating and inertial frames and the isotropic filter must be used in physical space such that

$$
\bar{u}_{i}(x)=\int G(|x-y|) u_{i}(y) \mathrm{d} y .
$$

The filtered equation of (2.8) and (2.9) can be written readily as

$$
\begin{gathered}
\frac{\partial \bar{u}_{i}}{\partial t}+U \frac{\partial \bar{u}_{i}}{\partial x_{1}}+\bar{u}_{k} \frac{\partial \bar{u}_{i}}{\partial x_{k}}=-\frac{1}{\rho} \frac{\partial \bar{p}}{\partial x_{i}}+v \frac{\partial^{2} \bar{u}_{i}}{\partial x_{k} \partial x_{k}}+\bar{s}_{i}+\frac{\partial \bar{\tau}_{i k}}{\partial x_{k}}, \\
\frac{\partial \bar{u}_{i}}{\partial x_{i}}=0,
\end{gathered}
$$

in which $\bar{\tau}_{i j}=\bar{u}_{i} \bar{u}_{j}-\overline{u_{i} u_{j}}$ is the subgrid stress. Note that the source term $s_{i}$ is a linear function of velocity fluctuations; hence, $\bar{s}_{i}$ is a linear function of filtered velocity fluctuations too, i.e. $\bar{s}_{i}=-\gamma \bar{u}_{2} \delta_{i 1}$ for homogeneous shear turbulence and $\bar{s}_{i}=2 \varepsilon_{i k 3} \bar{u}_{k} \Omega$ for homogeneous rotating turbulence. To obtain the Kolmogorov equation of resolved scale fluctuations, we require the momentum equation at a different spatial point $\boldsymbol{x}^{\prime}$,

$$
\frac{\partial \bar{u}_{i}^{\prime}}{\partial t}+U^{\prime} \frac{\partial \bar{u}_{i}^{\prime}}{\partial x_{1}^{\prime}}+\bar{u}_{k}^{\prime} \frac{\partial \bar{u}_{i}^{\prime}}{\partial x_{k}^{\prime}}=-\frac{1}{\rho} \frac{\partial \bar{p}^{\prime}}{\partial x_{i}^{\prime}}+v \frac{\partial^{2} \bar{u}_{i}^{\prime}}{\partial x_{k}^{\prime} \partial x_{k}^{\prime}}+\bar{s}_{i}^{\prime}+\frac{\partial \bar{\tau}_{i k}^{\prime}}{\partial x_{k}^{\prime}} .
$$

By subtraction of (2.12)from (2.10), a dynamic equation of velocity increment can be obtained as follows:

$$
\begin{aligned}
\frac{\partial \delta \bar{u}_{i}}{\partial t}+U \frac{\partial \bar{u}_{i}}{\partial x_{1}}-U^{\prime} \frac{\partial \bar{u}_{i}^{\prime}}{\partial x_{1}^{\prime}}+\bar{u}_{k} \frac{\partial \bar{u}_{i}}{\partial x_{k}}-\bar{u}_{k}^{\prime} \frac{\partial \bar{u}_{i}^{\prime}}{\partial x_{k}^{\prime}}=-\frac{1}{\rho} \frac{\partial \bar{p}}{\partial x_{i}}+\frac{1}{\rho} \frac{\partial \bar{p}^{\prime}}{\partial x_{i}^{\prime}} \\
+v \frac{\partial^{2} \bar{u}_{i}}{\partial x_{k} \partial x_{k}}-v \frac{\partial^{2} \bar{u}_{i}^{\prime}}{\partial x_{k}^{\prime} \partial x_{k}^{\prime}}+\bar{s}_{i}-\bar{s}_{i}^{\prime}+\frac{\partial \bar{\tau}_{i k}}{\partial x_{k}}-\frac{\partial \bar{\tau}_{i k}^{\prime}}{\partial x_{k}^{\prime}} .
\end{aligned}
$$

Note that $u_{i}$ is independent of $x_{i}^{\prime}$ and $u_{i}^{\prime}$ is independent of $x_{i}$, hence $\partial \bar{u}_{i} / \partial x_{k}^{\prime}=0$ and $\partial \bar{u}_{i}^{\prime} / \partial x_{k}=0$. The equation of velocity increment can then be rewritten as

$$
\begin{aligned}
& \frac{\partial \delta \bar{u}_{i}}{\partial t}+U \frac{\partial \delta \bar{u}_{i}}{\partial x_{i}}+U^{\prime} \frac{\partial \delta \bar{u}_{i}}{\partial x_{i}^{\prime}}+\bar{u}_{k} \frac{\partial \delta \bar{u}_{i}}{\partial x_{k}}+\bar{u}_{k}^{\prime} \frac{\partial \delta \bar{u}_{i}}{\partial x_{k}^{\prime}} \\
& =-\frac{1}{\rho} \frac{\partial \bar{p}}{\partial x_{i}}+\frac{1}{\rho} \frac{\partial \bar{p}^{\prime}}{\partial x_{i}^{\prime}}+v \frac{\partial^{2} \delta \bar{u}_{i}}{\partial x_{k} \partial x_{k}}+v \frac{\partial^{2} \delta \bar{u}_{i}}{\partial x_{k}^{\prime} \partial x_{k}^{\prime}}+\bar{s}_{i}-\bar{s}_{i}^{\prime}+\frac{\partial \bar{\tau}_{i k}}{\partial x_{k}}-\frac{\partial \bar{\tau}_{i k}^{\prime}}{\partial x_{k}^{\prime}},
\end{aligned}
$$

in which the velocity increment is defined as $\delta \bar{u}_{i}=\bar{u}_{i}-\bar{u}_{i}^{\prime}$. Multiplying the above equation by $\delta \bar{u}_{i}$, it becomes

$$
\begin{aligned}
& \frac{\partial\left(\delta \bar{u}_{i} \delta \bar{u}_{i}\right)}{\partial t}+U \frac{\partial\left(\delta \bar{u}_{i} \delta \bar{u}_{i}\right)}{\partial x_{i}}+U^{\prime} \frac{\partial\left(\delta \bar{u}_{i} \delta \bar{u}_{i}\right)}{\partial x_{i}^{\prime}}+\bar{u}_{k} \frac{\partial\left(\delta \bar{u}_{i} \delta \bar{u}_{i}\right)}{\partial x_{k}}+\bar{u}_{k}^{\prime} \frac{\partial\left(\delta \bar{u}_{i} \delta \bar{u}_{i}\right)}{\partial x_{k}^{\prime}} \\
& \quad=2\left(\frac{-\delta \bar{u}_{i}}{\rho} \frac{\partial \bar{p}}{\partial x_{i}}+\frac{\delta \bar{u}_{i}}{\rho} \frac{\partial \bar{p}^{\prime}}{\partial x_{i}^{\prime}}+v \delta \bar{u}_{i} \frac{\partial^{2} \delta \bar{u}_{i}}{\partial x_{k} \partial x_{k}}+v \delta \bar{u}_{i} \frac{\partial^{2} \delta \bar{u}_{i}}{\partial x_{k}^{\prime} \partial x_{k}^{\prime}}+\delta \bar{u}_{i} \delta \bar{s}_{i}+\delta \bar{u}_{i} \frac{\partial \bar{\tau}_{i k}}{\partial x_{k}}-\delta \bar{u}_{i} \frac{\partial \bar{\tau}_{i k}^{\prime}}{\partial x_{k}^{\prime}}\right) .
\end{aligned}
$$


The derivative of statistical correlations in respect to $\boldsymbol{x}$ or $\boldsymbol{x}^{\prime}$ can be transferred to the derivatives in respect to the displacement $\boldsymbol{\xi}=\boldsymbol{x}-\boldsymbol{x}^{\prime}$ in homogeneous turbulence as follows

$$
\frac{\partial}{\partial x_{i}}=\frac{\partial}{\partial \xi_{i}}, \quad \frac{\partial}{\partial x_{i}^{\prime}}=-\frac{\partial}{\partial \xi_{i}}, \quad \frac{\partial^{2}}{\partial x_{i} \partial x_{i}}=\frac{\partial^{2}}{\partial x_{i}^{\prime} \partial x_{i}^{\prime}}=\frac{\partial^{2}}{\partial \xi_{i} \partial \xi_{i}} .
$$

Taking an ensemble average on (2.13) and using the above derivation formulae, the terms in (2.13) can be simplified. The first term of the above equation is simply the time derivation of structure function $D_{i i}=\left\langle\delta \bar{u}_{i} \delta \bar{u}_{i}\right\rangle$, i.e. $\partial D_{i i} / \partial t$.

Since $U$ varies in the $x_{2}$-direction only, the sum of second and third terms on the left-hand side of (2.13) is

$$
\begin{array}{r}
\left\langle U \frac{\partial\left(\delta \bar{u}_{i} \delta \bar{u}_{i}\right)}{\partial x_{1}}\right\rangle+\left\langle U^{\prime} \frac{\partial\left(\delta \bar{u}_{i} \delta \bar{u}_{i}\right)}{\partial x_{1}^{\prime}}\right\rangle=\frac{\partial}{\partial \xi_{1}}\left\langle U \delta \bar{u}_{i} \delta \bar{u}_{i}\right\rangle-\frac{\partial}{\partial \xi_{1}}\left\langle U^{\prime} \delta \bar{u}_{i} \delta \bar{u}_{i}\right\rangle \\
=\frac{\partial}{\partial \xi_{1}}\left\langle\delta U \delta \bar{u}_{i} \delta \bar{u}_{i}\right\rangle=\delta U \frac{\partial D_{i i}}{\partial \xi_{1}}
\end{array}
$$

in which $\delta U=\gamma \xi_{2}$ in homogeneous shear turbulence and $\delta U=0$ in homogeneous rotating turbulence. The sum of the fourth and fifth terms on the left-hand side is

$$
\begin{aligned}
&\left\langle\frac{\partial \bar{u}_{k} \delta \bar{u}_{i} \delta \bar{u}_{i}}{\partial x_{k}}+\frac{\partial \bar{u}_{k}^{\prime} \delta \bar{u}_{i} \delta \bar{u}_{i}}{\partial x_{k}^{\prime}}\right\rangle=\frac{\partial}{\partial \xi_{k}}\left\langle\bar{u}_{k} \delta \bar{u}_{i} \delta \bar{u}_{i}\right\rangle-\frac{\partial}{\partial \xi_{k}}\left\langle\bar{u}_{k}^{\prime} \delta \bar{u}_{i} \delta \bar{u}_{i}\right\rangle \\
&=\frac{\partial}{\partial \xi_{k}}\left\langle\left(\bar{u}_{k}-\bar{u}_{k}^{\prime}\right) \delta \bar{u}_{i} \delta \bar{u}_{i}\right\rangle=\frac{\partial D_{i i k}}{\partial \xi_{k}}
\end{aligned}
$$

in which $D_{i i k}=\left\langle\delta \bar{u}_{k} \delta \bar{u}_{i} \delta \bar{u}_{i}\right\rangle$ is the third-order structure function. The terms involving pressure on the right-hand side can be simplified as

$$
\begin{gathered}
\left\langle\frac{\delta \bar{u}_{i}}{\rho} \frac{\partial \bar{p}}{\partial x_{i}}\right\rangle-\left\langle\frac{\delta \bar{u}_{i}}{\rho} \frac{\partial \bar{p}^{\prime}}{\partial x_{i}^{\prime}}\right\rangle=\frac{1}{\rho}\left\langle\frac{\partial \delta \bar{u}_{i} \delta \bar{p}}{\partial x_{i}}\right\rangle+\frac{1}{\rho}\left\langle\frac{\partial \delta \bar{u}_{i} \delta \bar{p}}{\partial x_{i}^{\prime}}\right\rangle-\frac{1}{\rho}\left\langle\bar{p} \frac{\partial \delta \bar{u}_{i}}{\partial x_{i}}\right\rangle \\
+\frac{1}{\rho}\left\langle\bar{p}^{\prime} \frac{\partial \delta \bar{u}_{i}^{\prime}}{\partial x_{i}^{\prime}}\right\rangle=\frac{1}{\rho} \frac{\partial\left\langle\delta \bar{u}_{i} \delta \bar{p}\right\rangle}{\partial \xi_{i}}-\frac{1}{\rho} \frac{\partial\left\langle\delta \bar{u}_{i} \delta \bar{p}\right\rangle}{\partial \xi_{i}}=0 .
\end{gathered}
$$

The homogeneity of turbulence and the continuity equation, i.e. $\partial \bar{u}_{i} / \partial x_{i}=0$ and $\partial \bar{u}_{i}^{\prime} / \partial x_{i}^{\prime}=0$, are used in the derivation of (2.16). It is not surprising that the pressure term is eliminated in the dynamic equation of $D_{i i}$, since pressure fluctuations contribute nothing to the energy transfer in incompressible homogeneous turbulence. The viscosity terms can be simplified as diffusive and dissipative terms such that

$$
\begin{gathered}
\left\langle\nu \delta \bar{u}_{i} \frac{\partial^{2} \delta \bar{u}_{i}}{\partial x_{k} \partial x_{k}}+v \delta \bar{u}_{i} \frac{\partial^{2} \delta \bar{u}_{i}}{\partial x_{k}^{\prime} \partial x_{k}^{\prime}}\right\rangle=\frac{v}{2} \frac{\partial^{2}\left\langle\left(\delta \bar{u}_{i}\right)^{2}\right\rangle}{\partial \xi_{k} \partial \xi_{k}}-\left\langle v \frac{\partial \delta \bar{u}_{i}}{\partial x_{k}} \frac{\partial \delta \bar{u}_{i}}{\partial x_{k}}\right\rangle \\
+\frac{v}{2} \frac{\partial^{2}\left\langle\left(\delta \bar{u}_{i}\right)^{2}\right\rangle}{\partial \xi_{k} \partial \xi_{k}}-\left\langle v \frac{\partial \delta \bar{u}_{i}}{\partial x_{k}^{\prime}} \frac{\partial \delta \bar{u}_{i}}{\partial x_{k}^{\prime}}\right\rangle=v \frac{\partial^{2} D_{i i}}{\partial \xi_{k} \partial \xi_{k}}-2 v\left\langle\frac{\partial \bar{u}_{i}}{\partial x_{k}} \frac{\partial \bar{u}_{i}}{\partial x_{k}}\right\rangle=v \frac{\partial^{2} D_{i i}}{\partial \xi_{k} \partial \xi_{k}}-2 \bar{\varepsilon}
\end{gathered}
$$

in which $\bar{\varepsilon}$ is the molecular dissipation of resolved scale of turbulence. The correlation between the difference of external force and velocity increment will be shown later, and the ensemble average of last two terms in (2.13) is expressed as follows

$$
\left\langle\delta u_{i}\left(\frac{\partial \bar{\tau}_{i k}}{\partial x_{k}}-\frac{\partial \bar{\tau}_{i k}^{\prime}}{\partial x_{k}^{\prime}}\right)\right\rangle=\left\langle\left(\frac{\partial \delta \bar{u}_{i} \bar{\tau}_{i k}}{\partial x_{k}}-\frac{\partial \delta \bar{u}_{i} \bar{\tau}_{i k}^{\prime}}{\partial x_{k}^{\prime}}\right)\right\rangle-\left\langle\left(\bar{\tau}_{i k} \frac{\partial \delta \bar{u}_{i}}{\partial x_{k}}-\bar{\tau}_{i k}^{\prime} \frac{\partial \delta \bar{u}_{i}}{\partial x_{k}^{\prime}}\right)\right\rangle .
$$


Now an eddy-viscosity assumption is introduced into the subgrid stress such that

$$
\bar{\tau}_{i k}=v_{t}\left(\frac{\partial \bar{u}_{i}}{\partial x_{k}}+\frac{\partial \bar{u}_{k}}{\partial x_{i}}\right)+\frac{1}{3} \bar{\tau}_{l l} \delta_{i k}
$$

in which $v_{t}$ is the subgrid eddy viscosity and is assumed to be a scalar constant in homogeneous turbulence. The last term of (2.19) has the same dynamic effect as pressure; hence, it is usually absorbed into the pressure term. Inserting (2.19) into (2.18), the simplification of the first two terms on the right-hand side of (2.18) can be derived as

$$
\begin{aligned}
\frac{\partial\left\langle\bar{\tau}_{i k} \delta \bar{u}_{i}\right\rangle}{\partial x_{k}}- & \frac{\partial\left\langle\bar{\tau}_{i k}^{\prime} \delta \bar{u}_{i}\right\rangle}{\partial x_{k}}=\frac{\partial\left\langle\bar{\tau}_{i k} \delta \bar{u}_{i}\right\rangle}{\partial \xi_{k}}+\frac{\partial\left\langle\bar{\tau}_{i k}^{\prime} \delta \bar{u}_{i}\right\rangle}{\partial \xi_{k}} \\
= & v_{t} \frac{\partial}{\partial \xi_{k}}\left\langle\left(\frac{\partial \bar{u}_{i}}{\partial x_{k}}+\frac{\partial \bar{u}_{k}}{\partial x_{i}}+\frac{\partial \bar{u}_{i}^{\prime}}{\partial x_{k}^{\prime}}+\frac{\partial \bar{u}_{k}^{\prime}}{\partial x_{i}^{\prime}}\right) \delta \bar{u}_{i}\right\rangle \\
= & v_{t} \frac{\partial}{\partial \xi_{k}}\left\langle\left(\frac{\partial \bar{u}_{i}}{\partial x_{k}}+\frac{\partial \bar{u}_{i}^{\prime}}{\partial x_{k}^{\prime}}\right) \delta \bar{u}_{i}\right\rangle+v_{t} \frac{\partial}{\partial \xi_{k}}\left\langle\left(\frac{\partial \bar{u}_{k}}{\partial x_{i}}+\frac{\partial \bar{u}_{k}^{\prime}}{\partial x_{i}^{\prime}}\right) \delta \bar{u}_{i}\right\rangle \\
= & v_{t} \frac{\partial}{\partial \xi_{k}}\left\langle\left(\frac{\partial\left(\bar{u}_{i}-\bar{u}_{i}^{\prime}\right)}{\partial x_{k}}+\frac{\partial\left(\bar{u}_{i}^{\prime}-\bar{u}_{i}\right)}{\partial x_{k}^{\prime}}\right) \delta u_{i}\right\rangle \\
& +2 v_{t} \frac{\partial^{2}\left\langle\delta \bar{u}_{k} \delta \bar{u}_{i}\right\rangle}{\partial \xi_{k} \partial \xi_{i}}=v_{t} \frac{\partial^{2}\left\langle\delta \bar{u}_{i} \delta \bar{u}_{i}\right\rangle}{\partial \xi_{i} \partial \xi_{i}}+2 v_{t} \frac{\partial^{2}\left\langle\delta \bar{u}_{k} \delta \bar{u}_{i}\right\rangle}{\partial \xi_{k} \partial \xi_{i}} .
\end{aligned}
$$

The last term of the above equation is equal to zero in homogeneous turbulence and it can be proved as follows

$$
\begin{aligned}
v_{t} \frac{\partial^{2}\left\langle\delta \bar{u}_{k} \delta \bar{u}_{i}\right\rangle}{\partial \xi_{k} \partial \xi_{i}} & =v_{t} \frac{\partial^{2}\left\langle\delta \bar{u}_{k} \delta \bar{u}_{i}\right\rangle}{\partial x_{k} \partial x_{i}}=v_{t}\left\langle\frac{\partial^{2}\left(\delta \bar{u}_{k} \delta \bar{u}_{i}\right)}{\partial x_{k} \partial x_{i}}\right\rangle \\
& =v_{t}\left\langle\frac{\partial \delta \bar{u}_{k}}{\partial x_{i}} \frac{\partial \delta \bar{u}_{i}}{\partial x_{k}}\right\rangle=v_{t}\left\langle\frac{\partial \bar{u}_{k}}{\partial x_{i}} \frac{\partial \bar{u}_{i}}{\partial x_{k}}\right\rangle=v_{t} \frac{\partial^{2}\left\langle\bar{u}_{k} \bar{u}_{i}\right\rangle}{\partial x_{k} \partial x_{i}} .
\end{aligned}
$$

Since the turbulence is homogeneous, the derivatives of one-point statistics are equal to zero, i.e.

$$
\frac{\partial^{2}\left\langle\bar{u}_{k} \bar{u}_{i}\right\rangle}{\partial x_{k} \partial x_{i}}=0
$$

and thus

$$
v_{t} \frac{\partial^{2}\left\langle\delta \bar{u}_{k} \delta \bar{u}_{i}\right\rangle}{\partial \xi_{k} \partial \xi_{i}}=v_{t} \frac{\partial^{2}\left\langle\bar{u}_{k} \bar{u}_{i}\right\rangle}{\partial x_{k} \partial x_{i}}=0 .
$$

The first two terms in (2.18) are then simplified as

$$
\frac{\partial\left\langle\bar{\tau}_{i k} \delta \bar{u}_{i}\right\rangle}{\partial x_{k}}-\frac{\partial\left\langle\bar{\tau}_{i k}^{\prime} \delta \bar{u}_{i}\right\rangle}{\partial x_{k}^{\prime}}=v_{t} \frac{\partial^{2}\left\langle\delta \bar{u}_{i} \delta \bar{u}_{i}\right\rangle}{\partial \xi_{i} \partial \xi_{i}}=v_{t} \frac{\partial^{2}\left\langle D_{i i}\right\rangle}{\partial \xi_{i} \partial \xi_{i}} .
$$

The last two terms of (2.18) are manipulated as follows

$$
\begin{gathered}
-\left\langle\left(\bar{\tau}_{i k} \frac{\partial \delta \bar{u}_{i}}{\partial x_{k}}-\bar{\tau}_{i k}^{\prime} \frac{\partial \delta \bar{u}_{i}}{\partial x_{k}^{\prime}}\right)\right\rangle=-v_{t}\left\langle\left(\frac{\partial \bar{u}_{i}}{\partial x_{k}}+\frac{\partial \bar{u}_{k}}{\partial x_{i}}\right) \frac{\partial \delta \bar{u}_{i}}{\partial x_{k}}\right\rangle+v_{t}\left\langle\left(\frac{\partial \bar{u}_{i}^{\prime}}{\partial x_{k}^{\prime}}+\frac{\partial \bar{u}_{k}^{\prime}}{\partial x_{i}^{\prime}}\right) \frac{\partial \delta \bar{u}_{i}}{\partial x_{k}^{\prime}}\right\rangle \\
=-v_{t}\left\langle\left(\frac{\partial \bar{u}_{i}}{\partial x_{k}}+\frac{\partial \bar{u}_{k}}{\partial x_{i}}\right) \frac{\partial \bar{u}_{i}}{\partial x_{k}}\right\rangle-v_{t}\left\langle\left(\frac{\partial \bar{u}_{i}^{\prime}}{\partial x_{k}^{\prime}}+\frac{\partial \bar{u}_{k}^{\prime}}{\partial x_{i}^{\prime}}\right) \frac{\partial \bar{u}_{i}^{\prime}}{\partial x_{k}^{\prime}}\right\rangle=-2 v_{t}\left\langle\frac{\partial \bar{u}_{i}}{\partial x_{k}} \frac{\partial \bar{u}_{i}}{\partial x_{k}}\right\rangle .
\end{gathered}
$$


In the above equation, $2 v_{t}\left\langle\left(\partial \bar{u}_{i} \partial x_{k}\right)\left(\partial \bar{u}_{i} \partial x_{k}\right)\right\rangle$, denoted as $\bar{\varepsilon}_{f}$, is the energy transfer from resolved scale to unresolved scale turbulence. Inserting those simplified expressions into the right-hand side of (2.18), it becomes

$$
\left\langle\delta u_{i}\left(\frac{\partial \bar{\tau}_{i k}}{\partial x_{k}}-\frac{\partial \bar{\tau}_{i k}^{\prime}}{\partial x_{k}^{\prime}}\right)\right\rangle=v_{t} \frac{\partial^{2}\left\langle\delta \bar{u}_{i} \delta \bar{u}_{i}\right\rangle}{\partial \xi_{i} \partial \xi_{i}}-2 v_{t}\left\langle\frac{\partial \bar{u}_{i}}{\partial x_{k}} \frac{\partial \bar{u}_{i}}{\partial x_{k}}\right\rangle=v_{t} \frac{\partial^{2} D_{l l}}{\partial \xi_{i} \partial \xi_{i}}+2 \bar{\varepsilon}_{f} .
$$

This shows clearly that subgrid stress in the dynamic equation of structure function plays the role of diffusion of structure function by eddy viscosity plus the transfer of turbulent kinetic energy from resolved scale to unresolved scale turbulence.

Take the ensemble average of (2.13) and insert all simplified expressions into it, the dynamic equation of structure function can be written as

$$
\frac{\partial D_{i i}}{\partial t}+\frac{\partial D_{i i k}}{\partial \xi_{k}}+\frac{\partial \delta U D_{i i}}{\partial \xi_{1}}=2\left(v+v_{t}\right) \frac{\partial^{2} D_{i i}}{\partial \xi_{k} \partial \xi_{k}}-4\left(v+v_{t}\right)\left\langle\frac{\partial \bar{u}_{i}}{\partial x_{k}} \frac{\partial \bar{u}_{i}}{\partial x_{k}}\right\rangle+2\left\langle\delta \bar{u}_{i} \delta \bar{s}_{i}\right\rangle .
$$

Equation (2.20) is the generalized Kolmogorov equation of anisotropic resolved scale turbulence. It is similar to the Kolmogorov equation for full turbulence without the eddy-viscosity term and the latter has been derived by Hill (2002). Following the classic theory that the time derivative of $D_{l l}$ and the molecular viscosity diffusion can be neglected in the dynamic equation of structure function for high-Reynolds-number flows (Monin \& Yaglom 1975), the final formula of the generalized Kolmogorov equation for anisotropic resolved scale turbulence is then written as

$$
\frac{\partial D_{i i k}}{\partial \xi_{k}}+\frac{\partial \delta U D_{i i}}{\partial \xi_{1}}=2 v_{t} \frac{\partial^{2} D_{i i}}{\partial \xi_{k} \partial \xi_{k}}-4 v_{t}\left\langle\frac{\partial \bar{u}_{i}}{\partial x_{k}} \frac{\partial \bar{u}_{i}}{\partial x_{k}}\right\rangle+2\left\langle\delta \bar{u}_{i} \delta \bar{s}_{i}\right\rangle .
$$

In order to obtain the eddy viscosity for homogeneous turbulence, we take the local volume average in displacement space as Hill (2002) and Casciola et al. (2004) did, as follows

$$
\int_{V}\left(\frac{\partial D_{i i k}}{\partial \xi_{k}}+\frac{\partial \delta U D_{i i}}{\partial \xi_{1}}\right) \mathrm{d} v=\int_{V}\left(2 v_{t} \frac{\partial^{2} D_{i i}}{\partial \xi_{k} \partial \xi_{k}}-4 v_{t}\left\langle\frac{\partial \bar{u}_{i}}{\partial x_{k}} \frac{\partial \bar{u}_{i}}{\partial x_{k}}\right\rangle+2\left\langle\delta \bar{u}_{i} \delta \bar{s}_{i}\right\rangle\right) \mathrm{d} v
$$

The volume integration on the left-hand side can be transferred to surface integration by the Gauss formula such that

$$
\int_{V} \frac{\partial D_{i i k}}{\partial \xi_{k}} \mathrm{~d} v=\int_{S} D_{i i k} n_{k} \mathrm{~d} A, \quad \int_{V} \frac{\partial \delta U D_{i i}}{\partial \xi_{1}} \mathrm{~d} v=\int_{S} \delta U D_{i i} n_{1} \mathrm{~d} A .
$$

The first term on right-hand side can also be transferred to surface integration such that

$$
\int_{V} 2 v_{t} \frac{\partial^{2} D_{i i}}{\partial \xi_{k} \partial \xi_{k}} d v=2 v_{t} \int_{S} \frac{\partial D_{i i}}{\partial \xi_{k}} n_{k} \mathrm{~d} A
$$

The linear size of the integration volume should be within the inertial subrange. In practice, it is equal to twice the mesh length in the following computational cases. Define the local volume average and surface average, respectively, as

$$
Q^{V}=\frac{1}{V} \int_{V} Q \mathrm{~d} v, \quad Q^{S}=\frac{1}{S} \int_{S} Q \mathrm{~d} A .
$$

Equation $(2.21 b)$ can be written as

$$
S\left(D_{i i k} n_{k}\right)^{S}+S\left(\delta U D_{i i} n_{1}\right)^{S}=S v_{t}\left(\frac{\partial D_{i i}}{\partial \xi_{k}} n_{k}\right)^{S}-4 v_{t} V\left\langle\frac{\partial \bar{u}_{i}}{\partial x_{k}} \frac{\partial \bar{u}_{i}}{\partial x_{k}}\right\rangle^{V}+2 V\left\langle\delta \bar{u}_{i} \delta \bar{s}_{i}\right\rangle^{V}
$$


Finally, the subgrid eddy viscosity is equal to

$$
v_{t}=\frac{\left(D_{i i k} n_{k}\right)^{S}+\left(\delta U D_{i i} n_{1}\right)^{S}+2 V / S\left\langle\delta \bar{u}_{i} \delta \bar{s}_{i}\right\rangle^{V}}{2\left(\frac{\partial D_{i i}}{\partial \xi_{k}} n_{k}\right)^{S}-4 V / S\left\langle\frac{\partial \bar{u}_{i}}{\partial x_{k}} \frac{\partial \bar{u}_{i}}{\partial x_{k}}\right\rangle^{V}} .
$$

The structure functions $D_{i i}=\left\langle\delta u_{i} \delta u_{i}\right\rangle, D_{i i k}=\left\langle\delta u_{i} \delta u_{i} \delta u_{k}\right\rangle$ are the ensemble average of the products of velocity increment in physical space, in practice the ensemble average can be taken in homogeneous directions; hence, the structure functions are independent of $\boldsymbol{x}$ and varying with displacement $\boldsymbol{\xi}$. The local volume average is taken in displacement space $\boldsymbol{\xi}$ which is independent of physical space $\boldsymbol{x}$. After local space averaging, the terms involving structure functions are constants both in physical and displacement spaces and, finally, the subgrid eddy viscosity is a constant in homogeneous turbulence. In practice, the local volume-average method is dependent on the flow geometry so that it can be taken in a sphere for homogeneous rotating turbulence or in a rectangular box for plane shear flow. The details of the average formulae will be given $\S \S 3$ and 4 .

In homogeneous rotating turbulence $\delta U=0$ and $\left\langle\delta \bar{u}_{i} \delta \bar{s}_{i}\right\rangle=2 \varepsilon_{i k 3}\left\langle\delta \bar{u}_{i} \delta \bar{u}_{k} \Omega\right\rangle$ where $\varepsilon_{i k 3}$ is an antisymmetrical tensor in respect to indices $i$ and $k$, hence $\left\langle\delta \bar{u}_{i} \delta \bar{s}_{i}\right\rangle=0$. The subgrid eddy viscosity for homogeneous rotating flow is then equal to

$$
v_{t}=\frac{\left(D_{i i k} n_{k}\right)^{S}}{2\left(\frac{\partial D_{i i}}{\partial \xi_{k}} n_{k}\right)^{S}-4 V / S\left\langle\frac{\partial \bar{u}_{i}}{\partial x_{k}} \frac{\partial \bar{u}_{i}}{\partial x_{k}}\right\rangle^{V}} .
$$

In homogeneous shear turbulence, $\delta U=\gamma \xi_{2}$ and $\left\langle\delta \bar{u}_{i} \delta \bar{s}_{i}\right\rangle=\gamma\left\langle\delta \bar{u}_{1} \delta \bar{u}_{2}\right\rangle$. The subgrid eddy viscosity becomes

$$
v_{t}=\frac{\left(D_{i i k} n_{k}\right)^{S}+\left(\gamma \xi_{2} D_{i i} n_{1}\right)^{S}-2 \gamma V / S\left\langle\delta \bar{u}_{1} \delta \bar{u}_{2}\right\rangle^{V}}{2\left(\frac{\partial D_{i i}}{\partial \xi_{k}} n_{k}\right)^{S}-4 V / S\left\langle\frac{\partial \bar{u}_{i}}{\partial x_{k}} \frac{\partial \bar{u}_{i}}{\partial x_{k}}\right\rangle^{V}} .
$$

Now, we would like to interpret the meaning of the subgrid eddy viscosity given above and make clear how the effect of anisotropy of turbulence is involved. In both equations (2.23) and (2.24), the numerator represents the transfer of velocity increment variance in resolved scale turbulence and the denominator represents the diffusion and dissipation of resolved scale turbulence by an eddy viscosity. In the inertial subrange, the transfer of turbulent kinetic energy must be equal to diffusion plus dissipation based on local equilibrium assumption. This is the radical property of our new model that involves the correct energy transfer between resolved and unresolved scale turbulence. Note that in spectral space, similar physical consideration leads to the definition of spectral subgrid eddy viscosity (Kraichnan 1976). In the case of homogeneous shear flow, the mean shear $\gamma$ takes part in the energy transfer, i.e. the second term and last terms in the numerator of (2.23), respectively. It clearly shows that the effect of mean shear on the eddy viscosity is not as simple as in the Smagorinsky model, i.e. $v_{t}=\left(C_{s} \Delta\right)^{2}|S|$, which will produce over-dissipation in the near-wall region. It will be shown later that the new model has correct asymptotic behaviour in the near-wall region, i.e. $v_{t} \propto x_{2}^{3}$ with $x_{2}$ in the normal direction to the wall.

In homogeneous rotating turbulence, the rotation rate does not appear explicitly in the formula of eddy viscosity, i.e. (2.24). It is not surprising because the solid 
rotation contributes nothing to the transfer of turbulent kinetic energy; neither does it to the transfer of velocity increment variance. However, rotation can redistribute kinetic energy among fluctuating velocity components and the turbulence becomes anisotropic. Equation (2.24) represents correct transfer of kinetic energy in anisotropic resolved scale turbulence through the local volume average of transfer and dissipation terms.

We will show that (2.22) can be simplified to the subgrid eddy viscosity in isotropic turbulence as derived in CZZS (2004). In isotropic turbulence, the transportation equation of structure function (2.21a) can be simplified as

$$
\frac{\partial D_{i i k}}{\partial \xi_{k}}=2 v_{t} \frac{\partial^{2} D_{i i}}{\partial \xi_{k} \partial \xi_{k}}-4 v_{t}\left\langle\frac{\partial \bar{u}_{i}}{\partial x_{k}} \frac{\partial \bar{u}_{i}}{\partial x_{k}}\right\rangle .
$$

Taking the local average in a sphere the in displacement space with radius $r$ in the inertial subrange, we obtain:

$$
\left(D_{i i r}\right)^{S_{r}}=2 v_{t}\left(\frac{\partial D_{i i}}{\partial r}\right)^{S_{r}}-\frac{4 v_{t}}{3}\left\langle\frac{\partial \bar{u}_{i}}{\partial x_{k}} \frac{\partial \bar{u}_{i}}{\partial x_{k}}\right\rangle^{V_{r}} r,
$$

in which $S_{r}=4 \pi r^{2}$ and $V_{r}=4 \pi r^{3} / 3$ so that $V_{r} / S_{r}=r / 3$. Following the derivation procedure by Hill (2002) it can be readily written as

$$
D_{l l l}=6 v_{t}\left(\frac{\partial D_{l l}}{\partial r}\right)^{S_{r}}-\frac{4 v_{t}}{5}\left\langle\frac{\partial \bar{u}_{i}}{\partial x_{k}} \frac{\partial \bar{u}_{i}}{\partial x_{k}}\right\rangle^{V_{r}} r
$$

which is the Kolmogorov equation for resolved scale isotropic turbulence at high Reynolds numbers. From (2.25), the subgrid eddy viscosity for isotropic turbulence can be written as (CZZS 2004)

$$
v_{t}=\frac{-5 D_{l l l}}{8\left\langle\bar{S}_{i j} \bar{S}_{i j}\right\rangle r-30 \partial D_{l l} / \partial r} .
$$

It is clear now that the previous subgrid eddy viscosity (CZZS 2004) is a simplified form of the new subgrid eddy viscosity for general homogeneous turbulence. The anisotropic effect on turbulent kinetic energy transfer is either revealed explicitly in homogeneous shear turbulence or included implicitly in homogeneous rotating flow by the use of the local volume average of the dynamic equation of structure function. We will show later that the new model improves the prediction of turbulent shear flows greatly in comparison with previous model (CZZS 2004) and can predict the major properties of rotating turbulence properly.

\section{Application to rotating turbulence}

Rotating turbulence is a typical case of anisotropic turbulence with practical interest in geophysical and astrophysical flows, as well as turbulent flows in turbomachinery. Considerable efforts have been made to investigate the behaviour of rotating turbulence and its structure by numerical simulation (Cambon, Mansour \& Godeferd 1997), experimental measurements (Jacquin et al. 1990) and theoretical analyses (Cambon, Rubinstein \& Godeferd 2004). A number of peculiar properties of rotating turbulence have been revealed so that the transfer of kinetic energy from large scales to small ones is reduced; consequently energy dissipation is decreasing with increasing rotating rates. It has also been found that the energy spectrum shows a steeper slope than the Kolmogorov -5/3 law so that the spectrum exponent 
approaches -3 asymptotically at infinite Reynolds number and zero Rossby number. It is obvious that the feature of rotating turbulence results from anisotropic nonlinear transfer of turbulent kinetic energy among velocity fluctuation components. The details of the behaviour of rotating turbulence can be found in (Cambon et al. 1997, 2004). In this paper, we are trying to simulate rotating turbulence numerically by LES. The traditional subgrid models fail to predict rotating turbulence since they do not consider the anisotropic effect of turbulence in the model. Yang \& Domaradski (2004) used truncated Navier-Stokes (TNS) as a model for large-eddy simulation of homogeneous rotating turbulence with considerable success; for instance, they obtained a $k^{-3}$ energy spectrum at high Reynolds numbers and small Rossby numbers. The idea of TNS is to try to model the energy transfer from large (non-truncated) to small-scale (truncated) turbulence by the estimation method. The new model presented here is different from theirs in that we formulate the eddy-viscosity model with the correct turbulent energy transfer from the Navier-Stokes equation without any assumptions on the velocity fluctuations, with the exception of homogeneity. Moreover, we will give more significant and critical statistical properties of rotating turbulence, such as the skewness of the velocity derivative, which was not given in Yang \& Domaradski (2004).

The numerical simulation of homogeneous rotating turbulence is performed in a rotating frame by the pseudo-spectrum method with rotation in the $x_{3}$-direction. The initial turbulence field is generated by the method proposed by Rogallo (1981) with a von Kármán spectrum. The computational domain is a rectangular box which is four times longer in the rotating direction than in the horizontal direction, since the turbulence scale is increasing greatly in the rotating direction. The fourth-order Runge-Kutta integration is used in time advancement. The time step is set to be small enough to resolve the inertial waves. The flow Reynolds number is assumed to be infinite by prescribing zero molecular viscosity.

In homogeneous rotating turbulence, the local average is taken in a sphere with radius $r$ and the subgrid eddy viscosity is derived from (2.25) so that

$$
v_{t}=\frac{3\left(D_{i i r}\right)^{S_{r}}}{6\left(\partial D_{i i} / \partial r\right)^{S_{r}}-4\left\langle\partial \bar{u}_{i} / \partial x_{k} \partial \bar{u}_{i} / \partial x_{k}\right\rangle^{V_{r} r}} .
$$

In practical computation, the local volume average is taken in a sphere circumscribing a cube with twice the mesh length.

The first test case is a decaying isotropic turbulence without rotation with $64^{3}$ grids in order to validate the model and numerical method. The simulation is run for more than $10^{6}$ initial turn-over time and the classic decaying law, $t^{-n}$, is found in a short time with $n \approx 1.25$, which is in agreement with previous numerical and experiment results. The exponent of the decaying law approaches -2 at the final stage of decay (figure 1), and this result is consistent with the experimental study by Skrbek \& Stalp (2000).

To perform the large-eddy simulation of decaying rotating turbulence at high Reynolds number, a purely decaying turbulence without rotation was computed for sufficient time and a solid-body rotation was then switched on when the time decay of large-scale kinetic energy $k$ reached a reliable power law in pre-computation. Figure 2 shows the time variation of the large-scale turbulence kinetic energy for different rotating rates. The numerical grid is $64 \times 64 \times 256$ in spectral space. As the rotation reduces the spectral energy transfer, the decay of the turbulent kinetic energy is becoming slower. This phenomenon is pronounced when the rotating rate is increasing. 


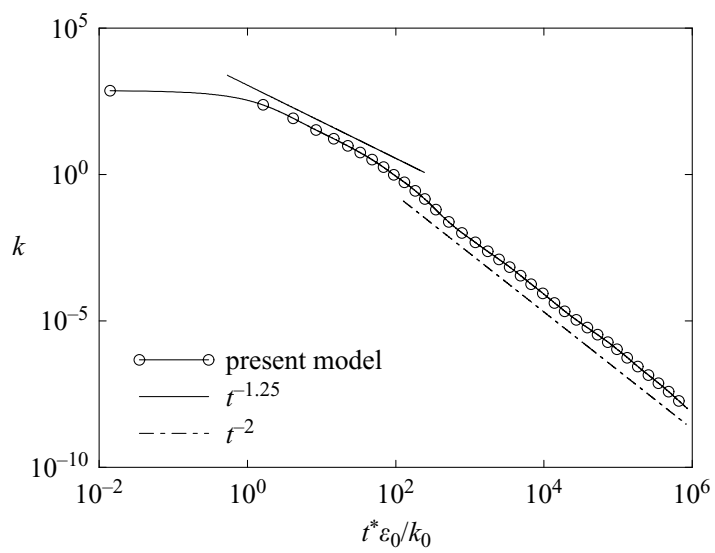

FIGURE 1. Decay of turbulent kinetic energy in isotropic turbulence with $64^{3}$ LES.

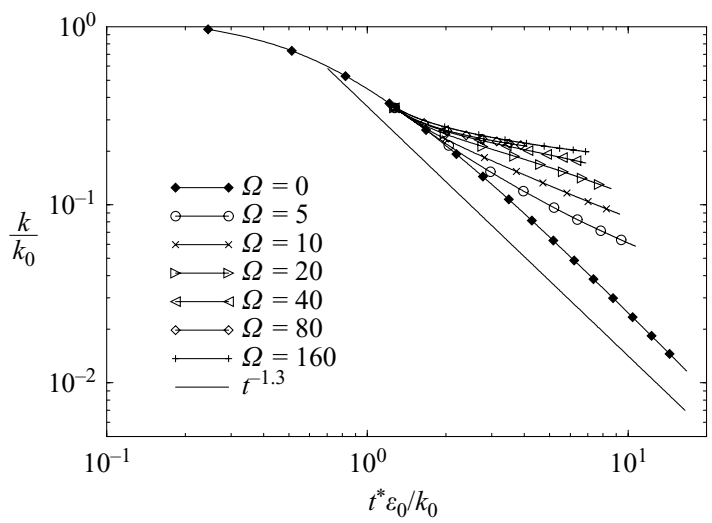

FIGURE 2. Time variation of large-scale kinetic energy for different rotating rates, grids: $64 \times 64 \times 256$

The critical examination of the feasibility of the new model is to check the variation of the derivative skewness with time. The derivative skewness, $S_{k}=\left\langle\left(\partial \bar{u} / \partial x_{1}\right)^{3}\right\rangle /\left\langle\left(\partial \bar{u} / \partial x_{1}\right)^{2}\right\rangle^{3 / 2}$, represents the balance between transfer of turbulent kinetic energy and its dissipation. The direct comparison of the derivative skewness between DNS and LES results is meaningless, but the variation of $S_{k} / S_{k 0}$ with time under the influence of rotation indicates the capability of the self-adjustment of a subgrid model to the rotation effect. In decaying turbulence, Cambon et al. (1997) proposed a scaling law of $S_{k} / S_{k 0}$ versus Rossby number, as follows:

$$
\frac{S_{k}(t)}{S_{k 0}}=\frac{1}{\left\{1+2\left[R o^{\omega}(t)\right]^{-2}\right\}^{0.5}},
$$

in which $R o^{\omega}=(\varepsilon / k \Omega) /\left(k^{2} / \nu \varepsilon\right)^{0.5}$ is based on the Taylor micro scale and called the micro Rossby number. In LES, the turbulent dissipation $\varepsilon$ and molecular viscosity $v$ are replaced by $\varepsilon_{f}$ and $v_{t}$, respectively, and $k$ is the large-scale turbulence kinetic energy. This replacement is valid so that the governing equation of LES for homogeneous turbulence with constant eddy viscosity is in a similar form to DNS with replacement of molecular viscosity by eddy viscosity. Following the same 


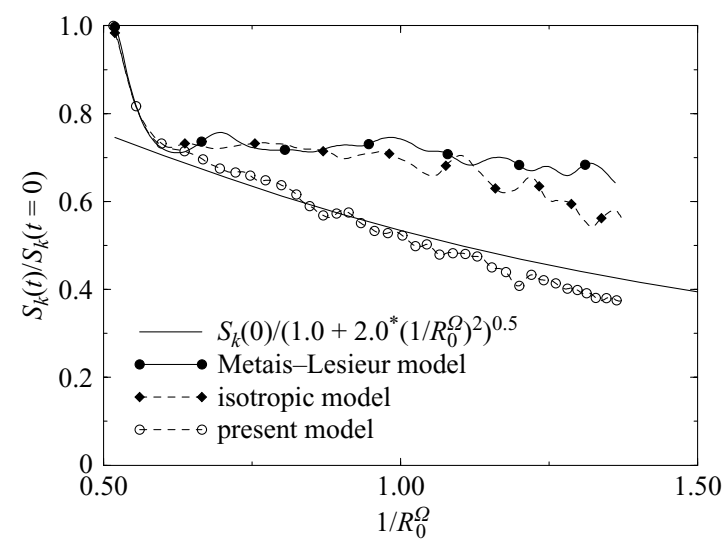

FIGURE 3. Skewness variation versus micro Rossby number at rotating rate $\Omega=10$.

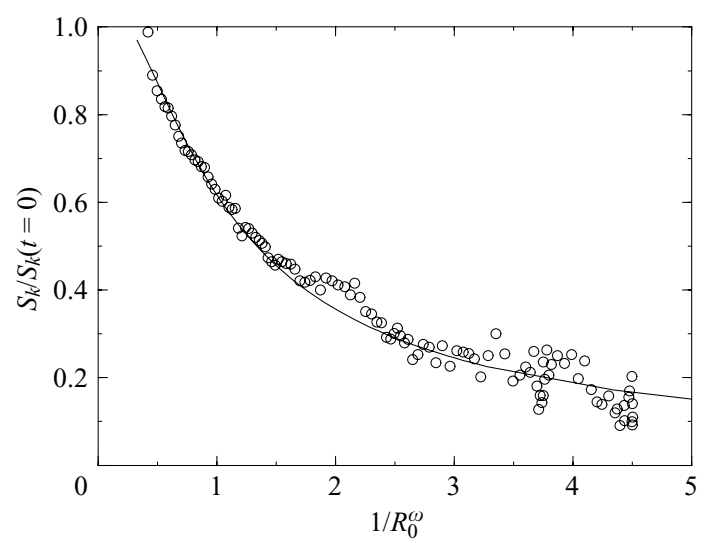

FIGURE 4. Skewness versus micro Rossby number with finer grids $96 \times 96 \times 384$ -, Cambon et al. (1997); O, anisotropic model.

deduction procedure as was performed by Cambon et al. (1997), it is reasonable to accept the above parameters in scale law (3.2). Comparison of the predicted results among different subgrid models is presented in figure 3 .

The initial micro Rossby number is equal to 1.82 at $\Omega=10$ and drops to 0.72 at the end of computation. All models produce a sudden reduction of derivative skewness to the level predicted by (3.2) at the beginning when rotation is applied and the Rossby number is relatively high. However, the later development differs greatly between the new model and the others. The Métais-Lesieur model (Métais \& Lesieur 1992) and the CZZS model cannot predict the reduction of skewness after a sudden reduction. The Métais-Lesieur mode predicts nearly constant level of skewness, whereas the CZZS model shows a small reduction of skewness. In contrast, the skewness predicted by the new model, which involves the anisotropic transfer of kinetic energy, fits the scaling law (3.2) very well, particularly at Rossby number around 1.

A finer grid with $96 \times 96 \times 384$ nodes was used to check the resolution influence. The micro Rossby number is nearly 2.0 in the initial state and reaches 0.22 at the end of computation. The result is shown in figure 4 and is much better than in the lower resolution run. 
(a)

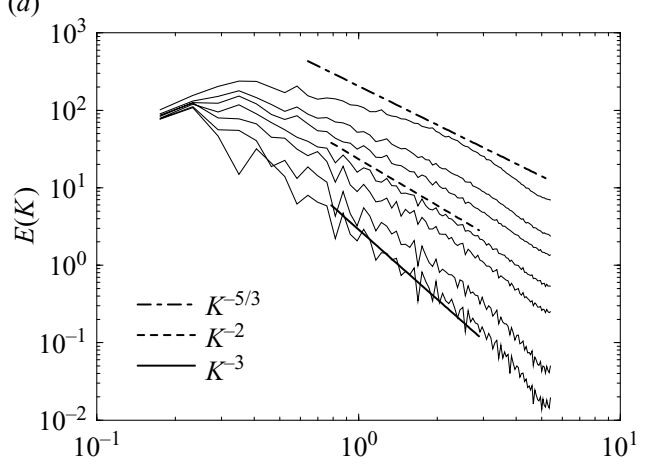

(c)

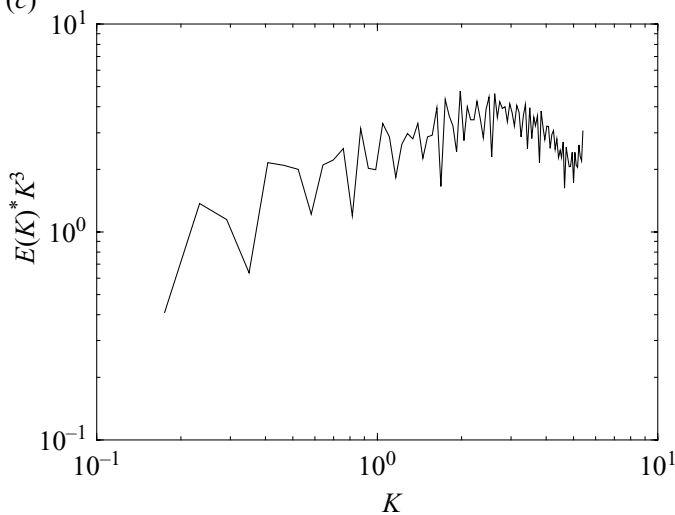

(b)

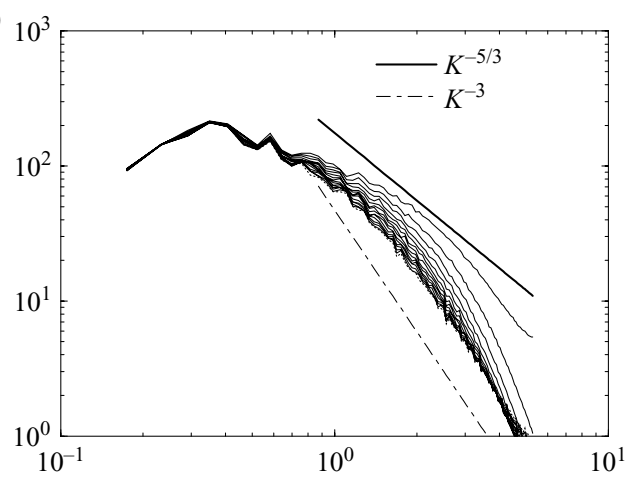

(d)

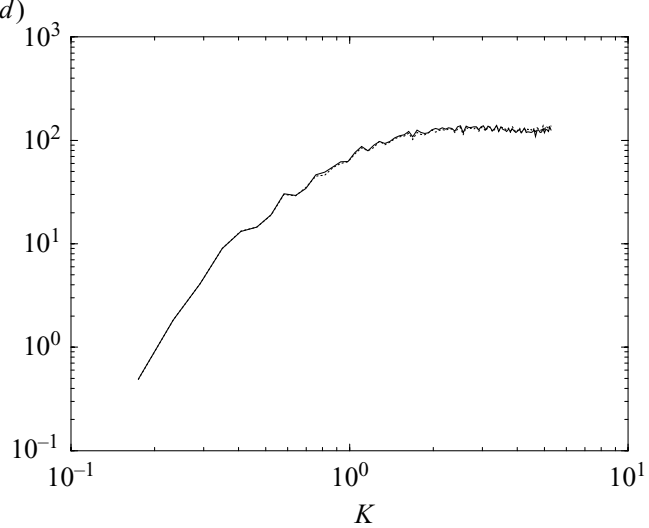

FIGURE 5. The evolution of energy spectrum with grids $96 \times 96 \times 384$. (a) $\Omega=10$; (b) $\Omega=100 ;(c)$ compensated $\Omega=10 ;(d)$ compensated $\Omega=100$.

The energy spectrum $E(k)$ is checked and presented in figure 5 for rotating rates of 10 and 100. The initial micro Rossby numbers are nearly 2.22 and 0.222 and approaching to 0.222 and 0.0667 at the end of computation. Once the rotation is switched on, the spectral slope shifts gradually from $-5 / 3$ to -3 as shown in figures $5(a)$ and $(b)$. In figures $5(c)$ and $5(d)$, the compensated spectra for both cases are plotted at the end of computation time. The plateau is shown clearly in both figures.

\section{Application to wall-bounded shear flows}

The proposed new model is based on the assumption of homogeneous turbulence. The homogeneity of turbulence can be accepted approximately in the major part of the wall-bounded turbulence, with the exception of the near-wall region. The proposed new model has correct asymptotic behaviour in the near-wall region so that $v_{t}$ is proportional to $y^{3}$ in the near-wall region; hence, the molecular viscosity is dominant there and the computation of wall-bounded turbulence can be performed by LES properly with fine normal resolution to the wall with the proposed new model. In fact, the previous CZZS model is capable of predicting turbulent channel flow in fairly good agreement with DNS results. Here, we will show that the proposed new model improves the prediction precision considerably.

In plane wall-bounded turbulent flows, the local volume average is taken in a rectangular element volume. The terms of local volume average, (2.22) can be 
manipulated as follows:

$$
\begin{aligned}
\left(D_{i i k} n_{k}\right)^{S}= & \frac{1}{S}\left(\iint_{S_{23}^{+}} D_{i i 1} \mathrm{~d} \xi_{2} \mathrm{~d} \xi_{3}-\iint_{S_{23}^{-}} D_{i i 1} \mathrm{~d} \xi_{2} \mathrm{~d} \xi_{3}+\iint_{S_{13}^{+}} D_{i i 2} \mathrm{~d} \xi_{1} \mathrm{~d} \xi_{3}\right. \\
& \left.-\iint_{S_{13}^{-}} D_{i i 2} \mathrm{~d} \xi_{1} \mathrm{~d} \xi_{3}+\iint_{S_{12}^{+}} D_{i i 3} \mathrm{~d} \xi_{1} \mathrm{~d} \xi_{2}-\iint_{S_{12}^{+}} D_{i i 3} \mathrm{~d} \xi_{1} \mathrm{~d} \xi_{2}\right) \\
\left(\frac{\partial D_{i i}}{\partial \xi_{k}} n_{k}\right)^{S}= & \frac{1}{S}\left(\iint_{S_{23}^{+}} \frac{\partial D_{i i}}{\partial \xi_{1}} \mathrm{~d} \xi_{2} \mathrm{~d} \xi_{3}-\iint_{S_{23}^{-}} \frac{\partial D_{i i}}{\partial \xi_{1}} \mathrm{~d} \xi_{2} \mathrm{~d} \xi_{3}+\iint_{S_{13}^{+}} \frac{\partial D_{i i}}{\partial \xi_{2}} \mathrm{~d} \xi_{1} \mathrm{~d} \xi_{3}\right. \\
& \left.-\iint_{S_{13}^{-}} \frac{\partial D_{i i}}{\partial \xi_{2}} \mathrm{~d} \xi_{1} \mathrm{~d} \xi_{3}+\iint_{S_{12}^{+}} \frac{\partial D_{i i}}{\partial \xi_{3}} \mathrm{~d} \xi_{1} \mathrm{~d} \xi_{2}-\iint_{S_{12}^{+}} \frac{\partial D_{i i}}{\partial \xi_{3}} \mathrm{~d} \xi_{1} \mathrm{~d} \xi_{2}\right),
\end{aligned}
$$

in which $S=2\left(\Delta \xi_{1} \Delta \xi_{2}+\Delta \xi_{1} \Delta \xi_{3}+\Delta \xi_{3} \Delta \xi_{2}\right)$. Since the turbulence is homogeneous in the $x_{1}$ - and $x_{3}$-directions in plane wall-bounded flows $\left(x_{2}\right.$ is assumed to be normal to the wall), two surface integrals on the element perpendicular to $x_{1}$, i.e. on $S_{23}^{+}$and $S_{23}^{-}$, cancel each other; the same results are obtained on $S_{12}^{+}$and $S_{12}^{-}$. Therefore, $\left(D_{i i k} n_{k}\right)^{S}$ and $\left(\left(\partial D_{i i} / \partial \xi_{k}\right) n_{k}\right)^{S}$ can be simplified as

$$
\begin{gathered}
\left(D_{i i k} n_{k}\right)^{S}=\frac{1}{S}\left(\iint_{S_{13}^{+}} D_{i i 2} \mathrm{~d} \xi_{1} \mathrm{~d} \xi_{3}-\iint_{S_{13}^{-1}} D_{i i 2} \mathrm{~d} \xi_{1} \mathrm{~d} \xi_{3}\right), \\
\left(\frac{\partial D_{i i}}{\partial \xi_{k}} n_{k}\right)^{S}=\frac{1}{S}\left(\iint_{S_{13}^{+}} \frac{\partial D_{i 2 i}}{\partial \xi_{2}} \mathrm{~d} \xi_{1} \mathrm{~d} \xi_{3}-\iint_{S_{13}^{-}} \frac{\partial D_{i i}}{\partial \xi_{2}} \mathrm{~d} \xi_{1} \mathrm{~d} \xi_{3}\right) .
\end{gathered}
$$

The integrals $\left(D_{i i k} n_{k}\right)^{S}$ and $\left(\left(\partial D_{i i} / \partial \xi_{k}\right) n_{k}\right)^{S}$ will be denoted by $\left[D_{i i 2}\right]^{A_{13}}$ and $\left[\partial D_{i i} / \partial \xi_{2}\right]^{A_{13}}$, respectively, hereinafter. The ratio of the local volume to its surface area is equal to

$$
\frac{V}{S}=\frac{\Delta x_{1} \Delta x_{2} \Delta x_{3}}{2\left(\Delta x_{1} \Delta x_{2}+\Delta x_{2} \Delta x_{3}+\Delta x_{1} \Delta x_{3}\right)}=\frac{\Delta x_{2}}{2\left(\Delta x_{2} / \Delta x_{3}+\Delta x_{2} / \Delta x_{1}+1\right)} .
$$

It can be expressed as $V / S=c_{y} \Delta x_{2}$ with $c_{y} \approx 0.5$ near the wall, and $\mathrm{c}_{y} \approx 1 / 6$ at the central part of channel the when we used the Gauss-Lobatto collocation in the normal direction. With similar manipulation of the other volume average terms, the subgrid eddy viscosity can be obtained as

$$
v_{t}=\frac{\left[D_{i i 2}\right]^{A_{13}}+2 \gamma\left(D_{12}\right)^{V} c_{y} \Delta_{y}}{\left[\partial D_{i i} / \partial \xi_{2}\right]^{A_{13}}-4\left\langle\partial u_{i} / \partial x_{k} \partial u_{i} / \partial x_{k}\right\rangle^{V} c_{y} \Delta_{y}},
$$

in which $\Delta_{y}=2 \Delta x_{2}$ is twice the local normal mesh length and $\gamma=\mathrm{d} U / \mathrm{d} y$ is the local mean strain rate. Since the magnitude of the velocity has asymptotic estimations at the wall in incompressible flows, as

$$
\bar{u}_{1} \propto y, \quad \bar{u}_{2} \propto y^{2}, \quad \bar{u}_{3} \propto y,
$$

it is easily to show that (4.4) leads to $v_{t} \propto y^{3}$ in the near-wall region, and this is correct asymptotic behaviour for subgrid stress. Note that the third and second structure functions are $D_{i i 2}$ and $D_{12}$ in the numerator of (4.4). This means that the kinetic energy is transferred by normal velocity fluctuations and it is a correct mechanism in the near-wall region.

The numerical method used in simulating turbulent plane Couette flow and channel flow is a pseudospectral method with Fourier decomposition in the $x_{1}$ - and 

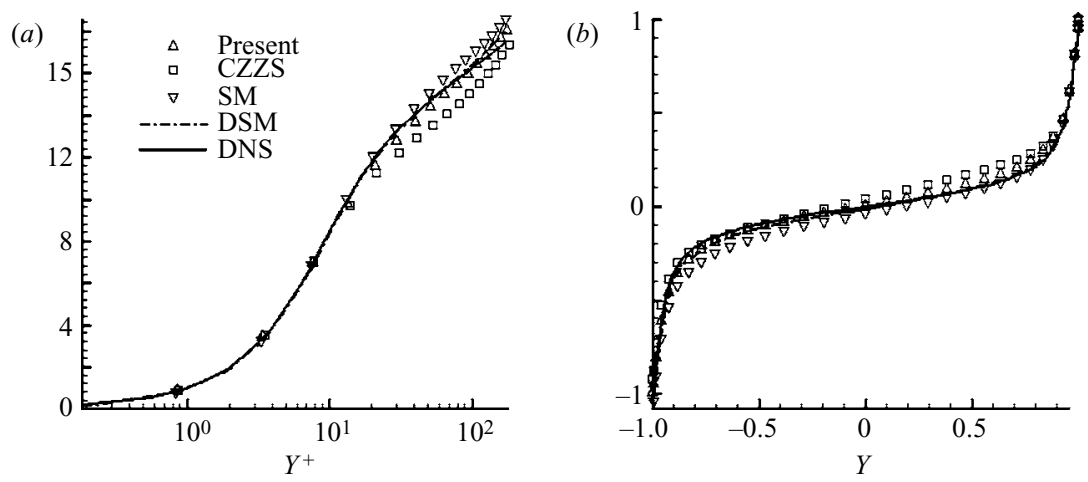

FIGURE 6. The mean velocity profile for plane Couette flow with grids $32 \times 64 \times 32$.

(a) $Y^{+}$scaled by wall units, (b) $Y$ scaled by $H$.

$x_{3}$-directions and Chebychev polynomial in the normal direction since the turbulence is homogeneous in the $x_{1}$ - and $x_{3}$-directions. The non-slip condition is posed at the wall without any modifications of models from wall effects, e.g. damping function or wall model. The third-order-accuracy scheme is used in time advancement. The computational domain is $2 H$ between two plates and $2 \pi H$ and $\pi H$ in the streamwise and spanwise direction, respectively. The details of the numerical method can be found in $\mathrm{Xu}$, Zhang \& Nieuwstadt (1997).

\subsection{The results of plane Couette flow}

The plane Couette flow is a good test case of the subgrid eddy-viscosity model for the homogeneous shear flow, since it has almost constant shear in the major part of the flow, apart from the near-wall region, which is a thin layer at high Reynolds number. The flow Reynolds number is defined as $U H / v$ in which $U$ is the moving speed of the upper plate and $R e=3200$ is accepted in the numerical simulation which is equivalent to a Reynolds number of 12800 in Kawamura, Abe \& Matsuo's DNS results (1999). The grid points are $32 \times 64 \times 32$ in the streamwise, normal and spanwise directions, respectively. The predicted mean velocity profile by the proposed new model is shown in figure 6 together with the results by DNS (Kawamura et al. 1999), the Smagorinsky model, the dynamic Smagroinsky model and the CZZS model (2004). In the computation with the Smagorinsky model, we use model coefficient $C_{s}=0.08$ and the van Driest damping function in the near-wall region.

Figure 7 shows the distribution of turbulent kinetic energy and Reynolds stress between two plates. In the plots, both turbulent kinetic energy and Reynolds stress include subgrid counterparts with the corrections given by Pope (2000). The results show that the new model is much better than the others.

\subsection{The results of turbulent plane channel}

Turbulent channel flow is another case for which to examine the feasibility of the proposed model. The turbulent channel is inhomogeneous in the direction normal to the wall; however, the mean shear rate is proportional to $1 / y^{+}$in the logarithm layer which is not so large, and the mean shear rate is much less above the logarithm layer. Therefore, the local homogeneity would be a good approximation in the major part of the channel flow. In the near-wall region, the model has the correct asymptotic behaviour as proved before, and it is expected that the proposed model is feasible in turbulent channel flows. 

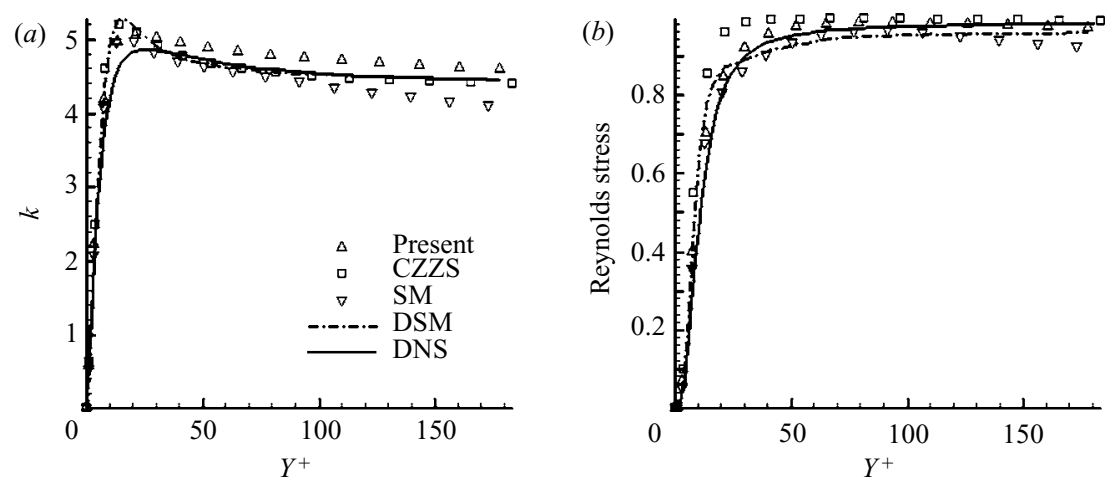

FIGURE 7. Distribution of turbulent statistics with grids $32 \times 64 \times 32$. (a) Turbulent kinetic energy $(b)$ Reynolds stress.
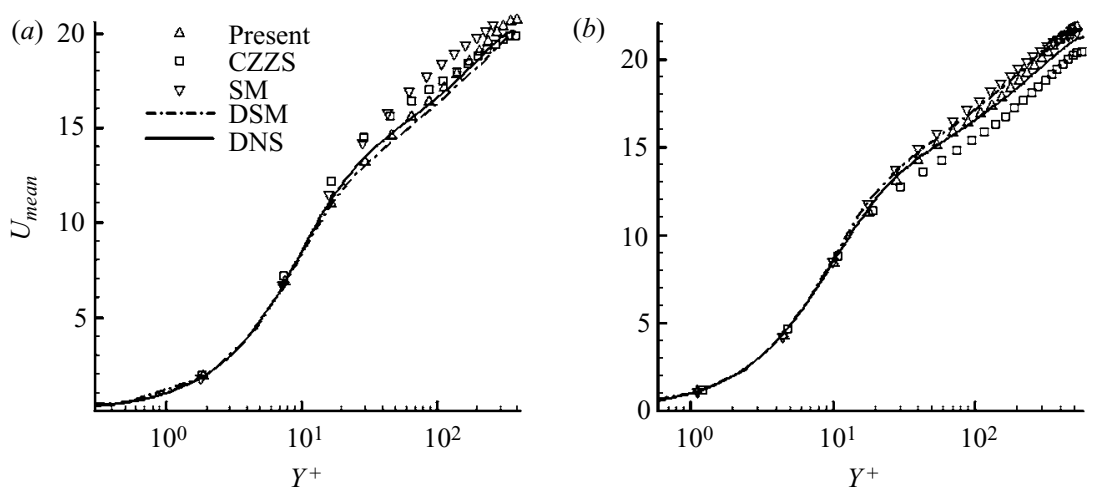

FIGURE 8 . The mean velocity profile with grids $(a) 32 \times 64 \times 32$ for $R e=7000$ and (b) $32 \times 96 \times 32$ for $R e=10000$.

The flow Reynolds number is defined as $U_{m} H / \nu$, in which $U_{m}$ is the bulk velocity in the channel and it is unchanged during the computation so that we use the constant-flow-rate condition in numerical simulation (Xu et al. 1997). Two test cases are computed at Reynolds numbers of 7000 and 10000 , which are equivalent to $R e_{\tau}=395,590$, respectively, in DNS performed by Moser, Kim \& Moin (1999) with constant pressure gradients. The grid points are $32 \times 64 \times 32$ in the steamwise, normal and spanwise directions, respectively, for $R e=7000$ and $32 \times 96 \times 32$ for $R e=10000$. Although our previous model (CZZS 2004) predicts fairly good statistics in turbulent channel flow, the new model gives much better results. This indicates that the inclusion of anisotropic transfer of turbulent kinetic energy is necessary and, indeed, improves the prediction.

Figure 8 presents the mean velocity profiles in which the prediction by the present subgrid model fits the DNS results well at both Reynolds numbers, and is better than previous model (CZZS 2004). Figure 9 shows the distribution of turbulent kinetic energy in which the correction of subgrid counterpart is added. The improvement of the present new model is evident; in particular, the location of the peak is close to that for the DNS results. Figure 10 shows the distribution of Reynolds stress in which the correction is also added. All results show that the proposed model is the best. 

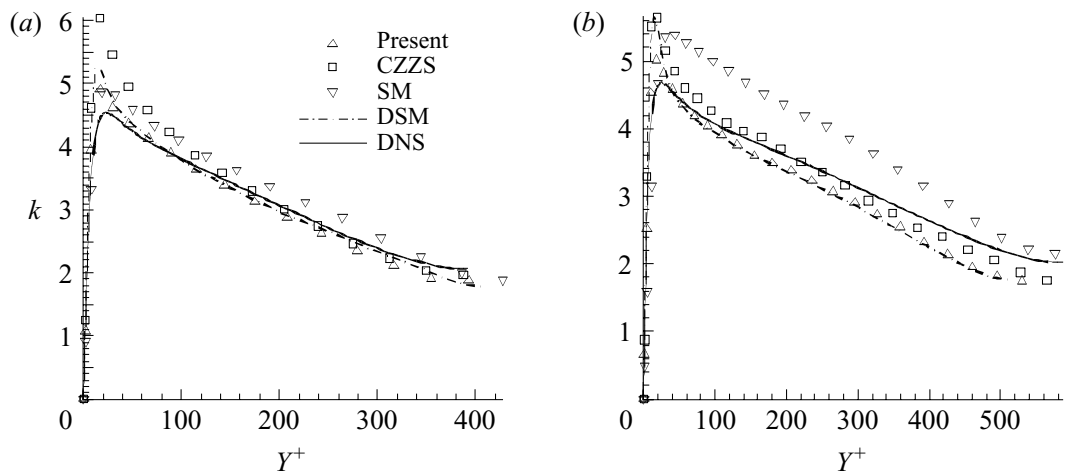

FIGURE 9. The turbulent kinetic energy with $(a)$ grids $32 \times 64 \times 32$ for $R e=7000$ and (b) $32 \times 96 \times 32$ for $R e=10000$.
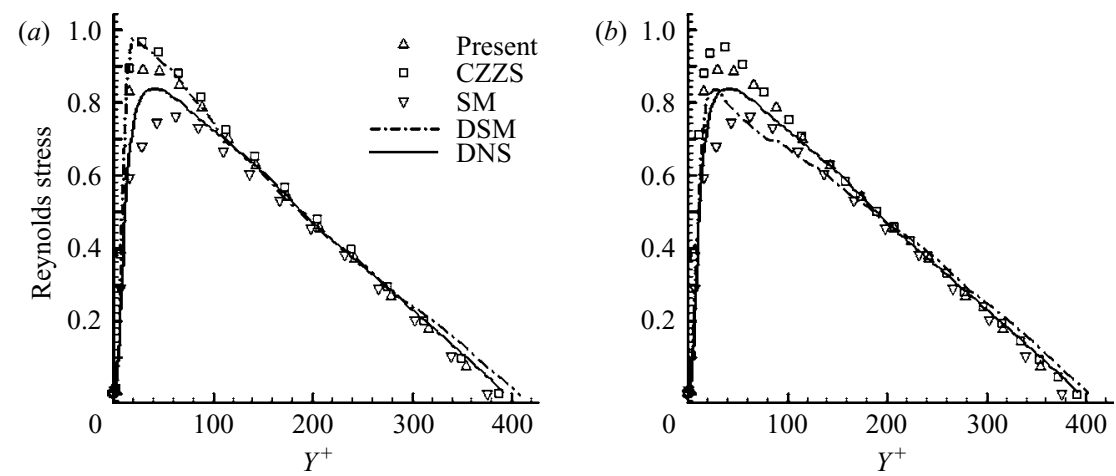

FIGURE 10. Reynolds stress with $(a)$ grids $32 \times 64 \times 32$ for $R e=7000$ and $(b) 32 \times 96 \times 32$ for $R e=10000$.

\section{Discussion and conclusions}

The new model has been examined in both homogeneous rotating turbulence and wall-bounded shear turbulence with satisfactory results. The success of this new type of subgrid model is attributed to the idea that the model is based on the turbulent energy transfer, which is derived directly from the Navier-Stokes equation without any phenomenological assumptions. This indicates that the model is consistent with the turbulent flow dynamics, and the subgrid eddy viscosity will be automatically adapted in different parts of a turbulent flow and in different turbulent flows.

The present model is established based on the homogeneity of turbulence, and is then applied to non-uniform shear turbulence. The extension of the homogeneous model, i.e. constant subgrid eddy viscosity, to non-uniform shear turbulence is reasonable when the flow can be considered to be locally homogeneous, and the eddy viscosity is accepted as locally constant. In a large part of the wall shear layer, the mean shear rates are small so that in the logarithm layer the mean shear rate is proportional to $1 / y^{+}$and it is much smaller above the logarithm layer. Therefore, the derivative of the mean shear rate is small and the local homogeneity can be acceptable. In the near-wall region, the proposed new model has correct asymptotic behaviour, i.e. $v_{t} \propto y^{3}$, and it can be used in the wall shear layer without any damping functions or wall models. The good predicted results from the present model in plane Couette and channel flows indicate that local constant subgrid eddy viscosity, which 


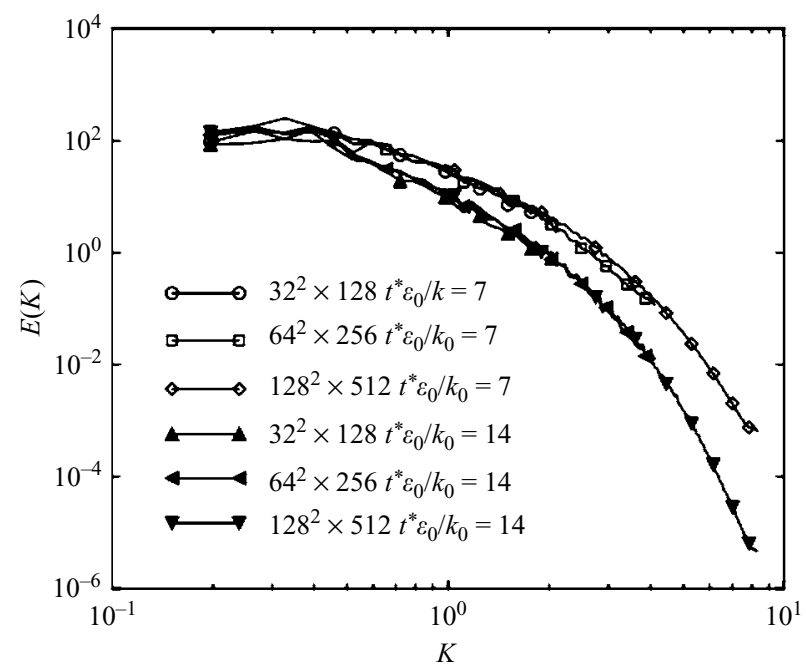

FIGURE 11. Comparison of energy spectrum between DNS $\left(128^{2} \times 512\right)$ and LES.

is a function of the normal distance to the wall, is acceptable, at least for the attached wall shear turbulence.

In rotating turbulence, the transformation property of the subgrid stress model should be concerned between the inertial frame and the rotating frame (Horiuti 2006). The present model is established for homogeneous rotating flow in a rotating frame by use of an isotropic filter and it can be proved that the generalized Kolmogorov equations for resolved scale turbulence of the homogeneous fluctuating motion are the same in both inertial and rotational frames. Therefore, the present subgridstress model satisfies the transformation property. In practice, some errors might be introduced by any slightly non-isotropic operations in numerical computation, but the errors are expected to be small, at least smaller than the modelling errors. To make sure that the influence of the practical numerical method on LES results is negligible, we compare the LES with DNS results at lower Renolds number $\operatorname{Re}_{\lambda}=50$ and rotating rate at $10 \mathrm{rad} \mathrm{s}^{-1}$. The comparison of energy spectrum is shown in figure 11 in which DNS is performed with grid number $128^{2} \times 512$ and LES is computed with coarse grids. The rotation is switched on at $t=2 k_{0} / \varepsilon_{0}=2$ and the energy spectra shown in figure 11 are at time $t=2 k_{0} / \varepsilon_{0}=7$ and 14 , respectively. The results of the LES are in good agreement with DNS.

In practical computation, the initial condition of velocity fluctuations is important in DNS and LES. For the new model, we need approximately correct initial structure functions, in particular $\left(D_{i i k} n_{k}\right)^{S}$. Numerical computation with an improper initial fluctuating field is bound to fail. There is no problem for homogeneous turbulence, since the initial structure function is approximately correct if we use some wellknown spectrum as the initial condition, e.g. Comte-Bellot spectrum or von-Kármán spectrum. During the time advancement, the spectrum will automatically evolve into the correct spectrum. For the wall-bounded turbulence, we have already checked from DNS and LES results that the skewness of the velocity increment, or the third-order structure function, is negative across the channel (CZZS 2004). Various ways can be applied to satisfy this condition in practical computation. One possible way is to use the data bank of lower-Reynolds-number cases as the initial condition for higherReynolds-flows in a channel with a non-dimensional correction. This is what we have 

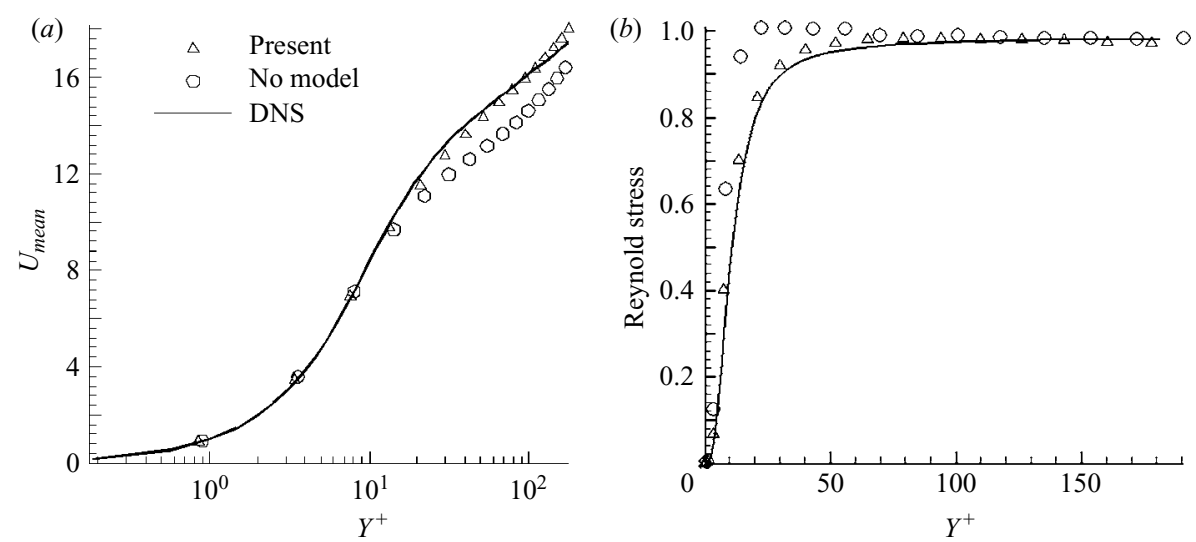

FiguRE 12. Comparison of the statistical properties of turbulent plane Couette flow between the new model and no model, $R e=3200$, grid $32 \times 64 \times 32$. (a) Mean velocity profiles. (b) Reynolds stress profiles.

done in test cases for turbulent plane Couette and channel flows. Another way is to start the computation by use of an easily accessible subgrid model, for instance the Smagorinsky model, and the new model will be switched on when the turbulent flow becomes nearly fully developed. We have tested this method and obtained results as good as by the first method. There may be other ways in practice, since the initial condition for LES, also in DNS, is a technical issue.

In numerical computation, the length $\Delta$ of the local average volume, equivalent to the filter length, is equal to twice the mesh lengths $h$ in the test cases. It has been proved that $\Delta / h=2$ is approximately optimum in numerical errors see (Geurts 2004; Pope 2004). For homogeneous rotating turbulence, the grid resolution depends on the turbulent Rossby number rather than on the Reynolds number. For instance, the $64 \times 64 \times 256$ grids are adequate for $R o^{\omega}<0.5$ whereas $98 \times 98 \times 384$ grids are marginally adequate for $R o^{\omega} \approx 0.2$ (figures 3 and 4 ). As far as the wall turbulent shear flows are concerned, higher spatial resolution is required in the wall-normal direction than in the horizontal directions for adequate simulation of the near-wall behaviours without wall model. For instance, 64 non-uniform grid points are enough for channel flow at $R e=7000$, whereas at least 96 non-uniform grid points are required at $R e=10000$. The requirement of resolution in streamwise and spanwise directions, i.e. in homogeneous directions, is not as serious as in normal direction, 32 uniform gird points are enough in LES, whereas at least 256 grid points are required in DNS for $R e=7000$ to 10000 . To check the effectiveness of the SGS stress in the computation, the comparison of the the predicted results between the new model and no model has been made (figures 12 and 13). The deviation of no model results is obvious from the DNS results in the mean velocity profiles and the deviation is great in the Reynolds stress.

The method used in derivation of subgrid eddy viscosity can be used to construct subgrid eddy diffusivity for scalar turbulence. A local volume average Yaglom equation is derived at first for filtered transportation equation of scalar turbulence, and the subgrid eddy diffusivity can be obtained as follows

$$
k_{t}=\frac{3\left(D_{r \theta \theta}\right)^{S_{r}}+3 \gamma r\left(n_{1} n_{2} D_{\theta \theta}\right)^{S_{r}}}{6 \frac{\mathrm{d}\left(D_{\theta \theta}\right)^{S_{r}}}{\mathrm{~d} r}-4\left\langle\frac{\partial \bar{\theta}}{\partial x_{k}} \frac{\partial \bar{\theta}}{\partial x_{k}}\right\rangle^{V_{r}} r},
$$



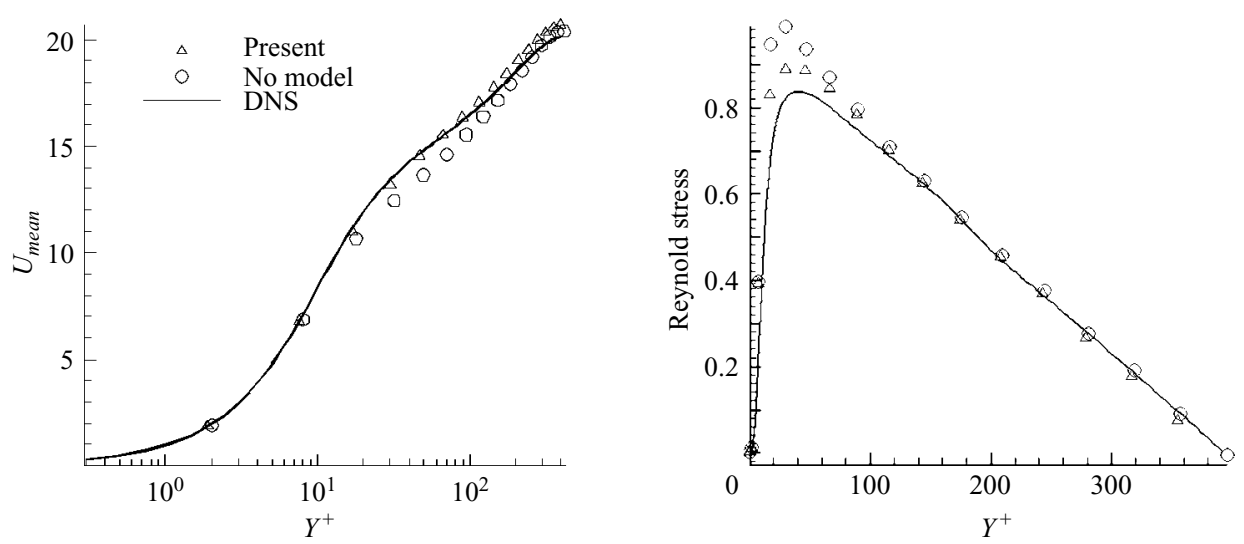

FIGURE 13. Comparison of the statistical properties of turbulent channel flow between the new model and no model, $R e=7000$, grid $32 \times 64 \times 32$. $(a)$ Mean velocity profiles. $(b)$ Reynolds stress profiles.

in which $D_{\theta \theta}$ is structure function for scalar turbulence, i.e. the variance of scalar increment, $D_{r \theta \theta}=\left\langle\left(u_{r}-u_{r}^{\prime}\right)\left(\theta-\theta^{\prime}\right)^{2}\right\rangle$ is the mixed third-order structure function. The application of new subgrid eddy diffusivity to homogeneous and wall-bounded turbulent flows is in progress and will be published later.

As far as the computational cost is concerned, the new model a little requires more computing time than the Smagorinsky model does but it costs much less than the dynamic Smagorinsky model. In turbulent channel flow, the computation cost is nearly 1:0.9:1.2 for the proposed new model, Smagorinsky model and dynamic Smagorinsky model, respectively.

In summary, the inclusion of the correct transfer of turbulent kinetic energy between resolved and unresolved scale turbulence is important in the construction of the subgrid stress model for large-eddy simulation. It can be realized in anisotropic turbulence by means of local volume-averaged generalized Kolmogorov equation for resolved scale turbulence. The new model has been tested successfully in rotating turbulence and wall-bounded turbulent flows.

The authors would like to thank Natural Science Foundation of China (Grant 10572073) and Sino-French Laboratory (LIAMA project 97-03) for financial supports. The authors are also grateful to the referees and Charles Meneveau for valuable comments.

\section{REFERENCES}

Bardina, J., Ferziger, J. \& Reynolds, W. C. 1987 Improved subgrid model for large-eddy simulation. AIAA Paper 80-1357.

Cambon, C., Mansour, N. N. \& Godeferd, F. S. 1997 Energy transfer in rotating turbulence. J. Fluid Mech. 337, 303.

Cambon, C., Rubinstein, R. \& Godeferd, F. S. 2004 Advances in wave turbulence: rapidly rotating flows. New J. Phys. 6, 73.

Casciola, C. M., Gualtieri, P., Benzi, R. \& Piva, R. 2004 Scale by scale budget and similarity law for shear turbulence. J. Fluid Mech. 476, 105.

Chollet, J. P. \& Lesieur, M. 1981 Parameterisation for small scales of three dimensional isotropic turbulence using spectral closure. J. Atmos. Sci. 38, 2747-2757. 
Cui, G. X., Zhou, H. B., Zhang, Z. S. \& ShaO, L. 2004 A new dynamic subgrid eddy viscosity model with application to turbulent channel flow. Phys. Fluids 16(8), 2835.

Gemano, M., Piomelli, U., Moin, P. \& Cавот, W. H. 1991 A dynamic subgrid-scale eddy viscosity model. Phys. Fluids, A3, 1760.

GeurTs, B. J. 2004 Elements of Direct and Large-Eddy Simulation. Edwards.

HiLl, R. J. 2002 Exact second-order structure function relationship. J. Fluid Mech. 468, 317.

HoRIUTI, K. 2006 Transformation properties of dynamic subgrid-scale models in a frame of reference undergoing rotation. J. Turbulence $7,1$.

Jacquin, L., Leuchter, O., Cambon, C. \& Mathieu, J. 1990 Homogeneous turbulence in the presence of rotation. J. Fluid Mech. 220, 1.

Jimenez, J. \& Moser, R. D. 2000 Large-eddy simulation: where are we and what can we expect? AIAA J. 38, 605.

Kawamura, H., Abe, H. \& Matsuo, Y. 1999 DNS of turbulent heat transfer in channel flow with respect to Reynolds and Prandtl number effects. Intl J. Heat Fluid Flow 20, 196-207.

Kraichnan, R. 1976 Eddy viscosity in two and three dimensions. J. Atmos. Sci. 1521-1536.

Meneveau, C. 1994 Statistics of turbulence subgrid-scale stress: necessary conditions and experimental tests. Phys. Fluids A 6, 815-833.

MÉtais, O. \& Lesieur, M. 1992 Spectral large-eddy simulation of isotropic and stably stratified turbulence. J. Fluid Mech. 256, 157-194.

Monin, A. S. \& Yaglom, A. M. 1975 Statistical Fluid Mechanics. Mechanics of Turbulence, Vol. 2. MIT Press.

Moser, R. D., Kim, J. \& Moin, P. 1999 Direct numerical simulation of turbulent channel flow up to $R e_{\tau}=590$. Phys Fluids 8, 1076.

Nicoud, F. \& Ducros, F. 1999 Subgrid-scale stress modeling based on the square of the velocity gradient tensor. Flow, Turbulence Combust 63, 183-200.

PoPe, S. 2004 Ten questions concerning the large eddy simulation of turbulent flows. New J. Phys. 6, 1-24.

Pope, S. 2000 Turbulent Flows. Cambridge University Press.

Rogallo, R. S. 1981 Numerical experiments in homogeneous turbulence. NASA Rep. 81315.

Sagaut, P. 2002 Large Eddy Simulation for Incompressible Flows. Springer.

Skrbek, L. \& Stalp, S. R. 2000 On the decay of homogeneous turbulence. Phys. Fluids 12(8), 1997.

SMAGORINSKY, J. 1963. General circulation experiments with primitive equation. Mon. Weather Rev. 91, 99.

VREMAN, A. W. 2004 An eddy-viscosity subgrid-scale mode for turbulent shear flow: algebraic theory and applications. Phys. Fluids 16, 3670-3681.

Xu, C., Zhang, Z. \& Nieuwstadt, F. T. M. 1996. Origin of high kurtosis in viscous sublayer. Phys. Fluids 8, 1938-1942.

YAng, X. \& Domaraski, J. A. 2004 Large eddy simulation of decaying turbulence. Phys. Fluids 16(11), 4088. 